\title{
Wigner density of a rigid rotator
}

\author{
C. P. Malta \\ Instituto de Física, Universidade de São Paulo, Caixa Postal 66318, 05315-970 São Paulo, Brazil \\ Trevor S. Marshall \\ Department of Mathematics, University of Manchester, Manchester M13 9PL, United Kingdom \\ Emilio Santos \\ Departamento de Física Moderna, Universidad de Cantabria, 39005, Santander, Spain
}

(Received 29 January 1996)

\begin{abstract}
We show that the Wigner density of the rigid rotator, in an appropriate, i.e., four-dimensional, phase space, is positive. This result holds in the ground state ( $S$ state), and also in the thermal mixture state at all finite temperatures. We discuss the implications of our result for the description of angular momentum in quantum mechanics; in particular, we reexamine, in the light of this new evidence, the suggestion made by Einstein and Stern [Ann. Phys. 40, 551 (1913)] that there is a nontrivial distribution of angular momentum in the $S$ state. [S1063-651X(97)11703-2]

PACS number(s): 05.30.- d, 03.65.Bz
\end{abstract}

\section{INTRODUCTION}

The Wigner density of a quantum-mechanical, oneparticle, zero-spin system, whose wave function is $\Psi(\mathbf{r})$, is $(\hbar=1)$

$$
W(\mathbf{p}, \mathbf{r})=\pi^{-3} \int \Psi^{*}(\mathbf{r}+\mathbf{R}) \Psi(\mathbf{r}-\mathbf{R}) e^{2 i \mathbf{p} \cdot \mathbf{R}} d^{3} \mathbf{R}
$$

The Wigner density is a pseudoprobability density; its integral over the momentum is the position density and vice versa, but, as a consequence of a general theorem $[1,2]$, it can be a positive function only for the very special case when $\Psi$ is Gaussian. So, according to these results, the only quantum-mechanical pure states which might be interpreted as probability distributions over the classical phase space are the squeezed coherent states of linear oscillators.

We shall show, in this paper, that the family may be enlarged, by a modest amount, to include a state of at least one nonlinear system, namely, the rigid rotator. The wave function of this state is not Gaussian, but we show that it nevertheless escapes the consequences of the above-mentioned theorem. This is because the nonpositivity of $W$ appears only in the density of the radial position and momentum. These coordinates, in the classically rigid case, would both be sharply defined, which would violate the Heisenberg relation. Actually a state with $r$ concentrated near some fixed value, $r_{0}$, will have a rather large range of values for $p_{r}$, but we shall see that the reduced Wigner density

$$
w\left(p_{\theta}, p_{\phi}, \theta, \phi\right)=\int_{0}^{\infty} d r \int_{-\infty}^{\infty} d p_{r} W(\mathbf{p}, \mathbf{r})
$$

is well defined for all pure quantum-mechanical states of the rigid rotator. Furthermore, its positivity properties are the same as those of the linear oscillator; it is positive in the ground state and, although it is not positive for the excited state, it is positive for all thermal mixtures. We see that the statistical mechanics of the rigid rotator in the Wigner representation is close to the classical statistical mechanics in the phase space.

A further consequence of the reduced Wigner description is that it enables us to reformulate the argument of Einstein and Stern [3], who saw, in the specific-heat curve of diatomic molecules, evidence in favor of a "hypothesis of molecular agitation at the absolute zero of temperature.' Einstein and Stern [3] obtained a remarkable agreement with the experimental data for the specific heat of diatomic molecules, using a simple model that included zero point fluctuations in the rotational energy. This agreement has been considered accidental by Milloni [4], but we shall show that it can be qualitatively explained in the Wigner representation.

We shall see that the joint Wigner density $V_{0}(\eta)$ of the three angular-momentum components $\left(\eta_{x}, \eta_{y}, \eta_{z}\right)$, in the state $\ell=0$, is not a $\delta$ function, but instead has dispersion

$$
\left\langle\eta_{x}^{2}+\eta_{y}^{2}+\eta_{z}^{2}\right\rangle_{0}=\int V_{0}(\eta) \eta^{2} d^{3} \eta=3 / 2 .
$$

This apparently paradoxical result is explained by a general property of the Wigner density that, for any observable $A(\eta)$ in the state $\Psi$,

$$
\langle\Psi|S \hat{A}| \Psi\rangle=\int A(\eta) V_{\Psi}(\eta) d^{3} \eta
$$

where $S$ denotes that the $\mathbf{r}$ and $\mathbf{p}$ operators of $\hat{A}$ have been symmetrically ordered. The modern argument [4] against Einstein and Stern is that, in the ground state $|0\rangle$,

$$
\hat{L}_{x}|0\rangle=\hat{L}_{y}|0\rangle=\hat{L}_{z}|0\rangle=0
$$

and hence

$$
\left\langle 0\left|\hat{L}_{x}^{2}+\hat{L}_{y}^{2}+\hat{L}_{z}^{2}\right| 0\right\rangle=0
$$


But it is easy to prove that

$$
S \hat{L}_{z}^{2}=S\left(\hat{x} \hat{p}_{y}-\hat{y} \hat{p}_{x}\right)^{2}=\hat{L}_{z}^{2}+1 / 2,
$$

and similarly for $L_{x}$ and $L_{y}$, which establishes the connection with Eq. (3).

This indicates that the zero point oscillations of $L_{x}, L_{y}$, and $L_{z}$ have the same character as those of the electromagnetic field, for which a single mode has the field energy $(\hbar=1)$,

$$
\hat{\epsilon}=\omega \hat{a}^{\dagger} \hat{a}
$$

where $\hat{a}$ is the photon annihilation operator in that mode. The mean square vacuum fluctuation of $\epsilon$ may be represented either by the normal ordering

$$
\Delta \epsilon_{N}^{2}=\left\langle 0\left|: \hat{\epsilon}^{2}:\right| 0\right\rangle-\langle 0|\hat{\epsilon}| 0\rangle^{2}=0
$$

or by the symmetric ordering

$$
\Delta \epsilon_{S}^{2}=\left\langle 0\left|S \hat{\epsilon}^{2}\right| 0\right\rangle-\langle 0|S \hat{\epsilon}| 0\rangle^{2}=\omega^{2} / 2 .
$$

Symmetrical ordering provides a picture closer to the classical one, and it has been advocated by Dalibard et al. [5]. However, the choice of a particular ordering to calculate expectation values is usually considered a matter of taste [6].

\section{THE REDUCED WIGNER DENSITY OF A SPHERICALLY SYMMETRIC SYSTEM}

The energy eigenfunctions of a spherically symmetric system are

$$
\Psi_{n \ell m}(\mathbf{r})=\psi_{n \ell}(r) Y_{\ell m}(\theta, \phi),
$$

where $Y_{\ell m}$ are normalized spherical harmonics and

$$
\int_{0}^{\infty} r^{2} \psi_{n \ell}^{2}(r) d r=1
$$

Putting Eq. (11) into Eq. (1) gives us a Wigner density $W_{n \ell m}(\mathbf{p}, \mathbf{r})$, but we immediately transfer attention to the mixed state, obtained by averaging over the magnetic quantum number,

$$
\bar{W}_{n \ell}(\mathbf{p}, \mathbf{r})=\frac{1}{2 \ell+1} \sum_{m=-\ell}^{\ell} W_{n \ell m}(\mathbf{p}, \mathbf{r}),
$$

for which the spherical harmonic addition theorem gives

$$
\begin{aligned}
\bar{W}_{n \ell}(\mathbf{p}, \mathbf{r})= & \frac{1}{8 \pi^{4} r} \int \psi_{n \ell}(u) \psi_{n \ell}(v) P \\
& \times\left(\frac{\mathbf{u} \cdot \mathbf{v}}{u v}\right) e^{2 i \mathbf{p} \cdot \mathbf{R}} u v d u d v d \chi
\end{aligned}
$$

where $\mathbf{u}=\mathbf{r}+\mathbf{R}, \mathbf{v}=\mathbf{r}-\mathbf{R}$, and $\chi$ is the angle between the plane containing $\mathbf{u}$ and $\mathbf{v}$ and the meridional plane through r.

Now put $p_{\text {tr }}=p_{\phi} \operatorname{cosec} \theta$; then

$$
\mathbf{p} \cdot \mathbf{R}=p_{r} \frac{\mathbf{r} \cdot \mathbf{R}}{r}+\left(p_{\theta} \cos \chi+p_{\mathrm{tr}} \sin \chi\right) \frac{|\mathbf{r} \times \mathbf{R}|}{r^{2}}
$$

and

$$
\begin{gathered}
\frac{\mathbf{r} \cdot \mathbf{R}}{r}=\frac{u^{2}-v^{2}}{4 r}, \\
\frac{|\mathbf{r} \times \mathbf{R}|}{r^{2}}=\left[\frac{u^{2}+v^{2}}{2 r^{2}}-1-\left(\frac{u^{2}-v^{2}}{4 r^{2}}\right)^{2}\right]^{1 / 2},
\end{gathered}
$$

so that

$$
\begin{aligned}
\int_{-\infty}^{\infty} e^{2 i \mathbf{p} \cdot \mathbf{R}} d p_{r}= & \frac{2 \pi r}{u} \delta(u-v) \\
& \times \exp \left[2 i\left(p_{\theta} \cos \chi+p_{\mathrm{tr}} \sin \chi\right) \sqrt{\frac{u^{2}}{r^{2}}-1}\right] .
\end{aligned}
$$

Hence

$$
\begin{aligned}
w_{n}\left(p_{\theta}, p_{\phi}, \theta, \phi\right)= & \frac{1}{4 \pi^{3}} \int_{0}^{\infty} d r \int_{r}^{\infty} d u \int_{0}^{2 \pi} d \chi^{\prime} u \psi_{n}^{2}(u) P_{\ell} \\
& \times\left(\frac{2 r^{2}-u^{2}}{u^{2}}\right) \exp \left[2 i \eta \cos \chi^{\prime} \sqrt{\frac{u^{2}}{r^{2}}-1}\right],
\end{aligned}
$$

where we have put

$$
\eta^{2}=p_{\theta}^{2}+p_{\mathrm{tr}}^{2} \text { and } \chi^{\prime}=\chi-\tan ^{-1} \frac{p_{\mathrm{tr}}}{p_{\theta}}
$$

Now put

$$
x=\sqrt{\frac{u^{2}}{r^{2}}-1} \cos \chi^{\prime} \text { and } y=\sqrt{\frac{u^{2}}{r^{2}}-1} \sin \chi^{\prime},
$$

then, using the normalization condition (12), we obtain

$$
\begin{aligned}
w_{\curlywedge}\left(p_{\theta}, p_{\phi}, \theta, \phi\right)= & \frac{1}{4 \pi^{3}} \int_{-\infty}^{\infty} d x \int_{-\infty}^{\infty} d y \frac{\exp (2 i \eta x)}{\left(1+x^{2}+y^{2}\right)^{3 / 2}} \\
& \times P\left(\frac{1-x^{2}-y^{2}}{1+x^{2}+y^{2}}\right)
\end{aligned}
$$

Note that this reduced Wigner density does not depend at all on the radial wave function; it is the same for all spherically symmetric systems and depends only on the total angular momentum quantum number $\ell$. The integrations in Eq. (22) may be done by introducing the generating function of $P_{\ell}$ in the form

$$
\begin{aligned}
& \sum(2 \ell+1) z^{\ell} P\left(\frac{1-t^{2}}{1+t^{2}}\right) \\
& \quad=\frac{1-z}{(1+z)^{2}}\left(1+t^{2}\right)^{3 / 2}\left[\left(\frac{1-z}{1+z}\right)^{2}+t^{2}\right]^{-\frac{3}{2}}
\end{aligned}
$$


which gives, on substituting into Eq. (22),

$$
\sum(2 \ell+1) z^{\ell} w=\frac{1}{2 \pi^{2}(1+z)} \exp \left(-2 \eta \frac{1-z}{1+z}\right),
$$

and hence

$$
w_{\ell}\left(p_{\theta}, p_{\phi}, \theta, \phi\right)=\frac{(-1)^{\ell}}{2 \pi^{2}(2 \ell+1)} L /(4 \eta) e^{-2 \eta},
$$

where $L_{\ell}$ are the Laguerre polynomials.

\section{ANGULAR MOMENTUM AS A CLASSICAL RANDOM VARIABLE}

The reduced Wigner density, obtained in the preceding section, is a function of the single variable

$$
\eta=\sqrt{p_{\theta}^{2}+p_{\mathrm{tr}}^{2}}=\sqrt{p_{\theta}^{2}+p_{\phi}^{2} \operatorname{cosec}^{2} \theta}
$$

which may be identified as the total angular momentum of the system. As may have been anticipated from the well known case of the linear oscillator, this density is positive only for the case $\ell=0$, but in view of the Hudson-SotoClaverie theorem $[1,2]$, it is a pleasant surprise, even in this case, to find that it is positive.

Now, using the pseudoprobabilities $w_{\ell}$ in the form of their generating function (24), it is straightforward to obtain the expectation value of powers of $\eta$, that is,

$$
\left\langle\eta^{n}\right\rangle_{\ell}=\int w_{\ell}\left(p_{\theta}, p_{\phi}, \theta, \phi\right) \eta^{n} d p_{\theta} d p_{\phi} d \theta d \phi
$$

We obtain

$$
\sum(2 \ell+1)\left\langle\eta^{n}\right\rangle z^{\ell}=2^{-n}(n+1) ! \frac{(1+z)^{n+1}}{(1-z)^{n+2}},
$$

and, in particular,

$$
\begin{aligned}
& \langle\eta\rangle_{\ell}=\frac{2 \ell^{2}+2 \ell+1}{2 \ell+1}, \\
& \left\langle\eta^{2}\right\rangle_{\ell}=\ell^{2}+\ell+3 / 2 .
\end{aligned}
$$

As we remarked in the Introduction, the second of these results may be obtained directly from the operator algebra, but the first, as far as we know, is new, and can be obtained only from the Wigner density. The two results together would, if the moments were true moments, give a "dispersion', for $\eta$ of

$$
\Delta \eta_{\ell}^{2}=\left\langle\eta^{2}\right\rangle_{\ell}-\langle\eta\rangle_{\ell}^{2}=\frac{3}{4}-\frac{1}{4(2 \ell+1)^{2}},
$$

which would indicate a distribution of $\eta$ just sufficiently concentrated to distinguish $w_{\ell}$ from $w_{\ell+1}$. Such a description is not altogether reliable, however, since a closer examination of $w_{\ell}$ reveals that its "negative-probability" oscillations extend well beyond the region defined by $\ell+\frac{1}{2}$ $\pm \Delta \eta_{\ell}$.

Nevertheless, we shall anticipate the results of the next section which, to a large extent, resolve the pseudoprobabil- ity problem, and exercise that freedom, which we have in classical statistical mechanics, to change the variables in phase space.

As a first step we shall change from the variables $\left(p_{\theta}, p_{\phi}, \theta, \phi\right)$ to $\left(p_{\theta}, p_{\text {tr }}, \theta, \phi\right)$, where

$$
p_{\mathrm{tr}}=p_{\phi} \operatorname{cosec} \theta
$$

In terms of these variables we have

$$
w_{\ell}\left(p_{\theta}, p_{\mathrm{tr}}, \theta, \phi\right)=\frac{(-1)^{\ell}}{2 \pi^{2}(2 \ell+1)} e^{-2 \eta} L_{\ell}(4 \eta) \sin \theta,
$$

where

$$
\eta=\sqrt{p_{\theta}^{2}+p_{\mathrm{tr}}^{2}}
$$

with the normalization

$$
\int w_{\ell}\left(p_{\theta}, p_{\mathrm{tr}}, \theta, \phi\right) d p_{\theta} d p_{\mathrm{tr}} d \theta d \phi=1
$$

This shows uniform distribution with respect to the solid angle $\sin \theta d \theta d \phi$, and, at the same time, isotropy with respect to the meridional angular momentum $p_{\theta}$ and the transverse angular momentum $p_{\text {tr }}$.

Now we introduce the Cartesian components $\left(\eta_{x}, \eta_{y}, \eta_{z}\right)$ of angular momentum, so that

$$
\eta=\sqrt{\eta_{x}^{2}+\eta_{y}^{2}+\eta_{z}^{2}}
$$

and we see that, after averaging over the surface of the sphere, the isotropy over $\left(p_{\theta}, p_{\text {tr }}\right)$ translates into one over $\left(\eta_{x}, \eta_{y}, \eta_{z}\right)$. We deduce that the reduced density with respect to $\eta$ is

$$
V /\left(\eta_{x}, \eta_{y}, \eta_{z}\right)=\frac{(-1)^{\ell} e^{-2 \eta}}{(2 \ell+1) \pi \eta} L /(4 \eta),
$$

with the normalization

$$
\int V_{\curlywedge}\left(\eta_{x}, \eta_{y}, \eta_{z}\right) d \eta_{x} d \eta_{y} d \eta_{z}=1
$$

This is the joint "probability" density over the variables corresponding to the noncommuting observables $\left(\hat{L}_{x}, \hat{L}_{y}, \hat{L}_{z}\right)$. In accordance with a general theorem on Wigner densities, it satisfies the relation

$$
\begin{aligned}
& \frac{1}{2 \ell+1} \sum_{m=-\ell}^{\ell}\left\langle\ell m\left|\mathrm{~S}\left(\hat{L}_{x}^{p} \hat{L}_{y}^{q} \hat{L}_{z}^{r}\right)\right| \ell m\right\rangle \\
& \quad=\int V \ell\left(\eta_{x}, \eta_{y}, \eta_{z}\right) \eta_{x}^{p} \eta_{y}^{q} \eta_{z}^{r} d \eta_{x} d \eta_{y} d \eta_{z} .
\end{aligned}
$$

\section{THE THERMODYNAMICS OF THE RIGID ROTATOR}

In the case of the rigid rotator, with its phase space restricted by the rigidity, the reduced Wigner density of Sec. II, that is, 


$$
\begin{gathered}
w \curlywedge\left(p_{\theta}, p_{\phi}, \theta, \phi\right)=\frac{(-1)^{\ell}}{2 \pi^{2}(2 \ell+1)} L(4 \eta) e^{-2 \eta}, \\
\eta=\sqrt{p_{\theta}^{2}+p_{\phi}^{2} \operatorname{cosec}^{2} \theta}
\end{gathered}
$$

becomes the full Wigner density corresponding to the angular momentum quantum number $\ell$. We note the close formal resemblance to the Wigner density of the one-dimensional linear oscillator in its $n$th excited state

$$
\begin{gathered}
W_{n}(p, x)=\frac{(-1)^{n}}{\pi} L_{n}(4 \eta) e^{-2 \eta} \\
\eta=(2 m \omega)^{-1}\left(p^{2}+m^{2} \omega^{2} x^{2}\right) .
\end{gathered}
$$

The problem of the nonpositivity of $W_{n}(p, x)$ has been extensively discussed, both in its thermodynamic [7] and spectroscopic [8] aspects. It has been shown that all laboratory states of the linear oscillator, both equilibrium and nonequilibrium, are, in fact, mixtures, represented by positive Wigner densities, of pure states. This result was established by representing the interaction of the oscillator with the electromagnetic field as a classical stochastic process, known as stochastic electrodynamics $[9,10]$. But such a representation is known to fail in the case of nonlinear systems [11]; it follows that we cannot carry out a comprehensive treatment of the rigid rotator, as we were able to do with the linear oscillator. Nevertheless, we shall show that all the equilibrium states of the rigid rotator, corresponding to thermal mixtures of the densities $w_{\ell}$, have positive Wigner densities.

The Wigner density at temperature $T$ [in units of $\left.\hbar^{2}(2 I k)^{-1}\right]$ is

$$
w\left(p_{\theta}, p_{\phi}, \theta, \phi ; T\right)=\frac{G(\eta ; T)}{4 \pi^{2} Z(T)}=\frac{F(\eta ; T)}{2 \pi^{2}}(\text { say })
$$

where

$$
G(\eta ; T)=2 \sum_{\ell=0}^{\infty}(-1)^{\ell} L_{\ell}(4 \eta) \exp \left(-2 \eta-\frac{\ell(\ell+1)}{T}\right),
$$

and $Z(T)$ is the partition function

$$
Z(T)=\sum_{\ell=0}^{\infty}(2 \ell+1) \exp \left(-\frac{\ell(\ell+1)}{T}\right)
$$

The alternating partial sums of the $L_{\ell}$ are defined as

$$
S_{n}(x)=L_{0}(x)-L_{1}(x)+L_{2}(x)-\cdots+(-1)^{n} L_{n}(x) .
$$

Then Eq. (45) may be written as

$$
\begin{aligned}
G(\eta ; T)= & 2 \sum_{n=0}^{\infty} S_{n}(4 \eta) e^{-2 \eta}\left[\exp \left(-\frac{n(n+1)}{T}\right)\right. \\
& \left.-\exp \left(-\frac{(n+1)(n+2)}{T}\right)\right]
\end{aligned}
$$

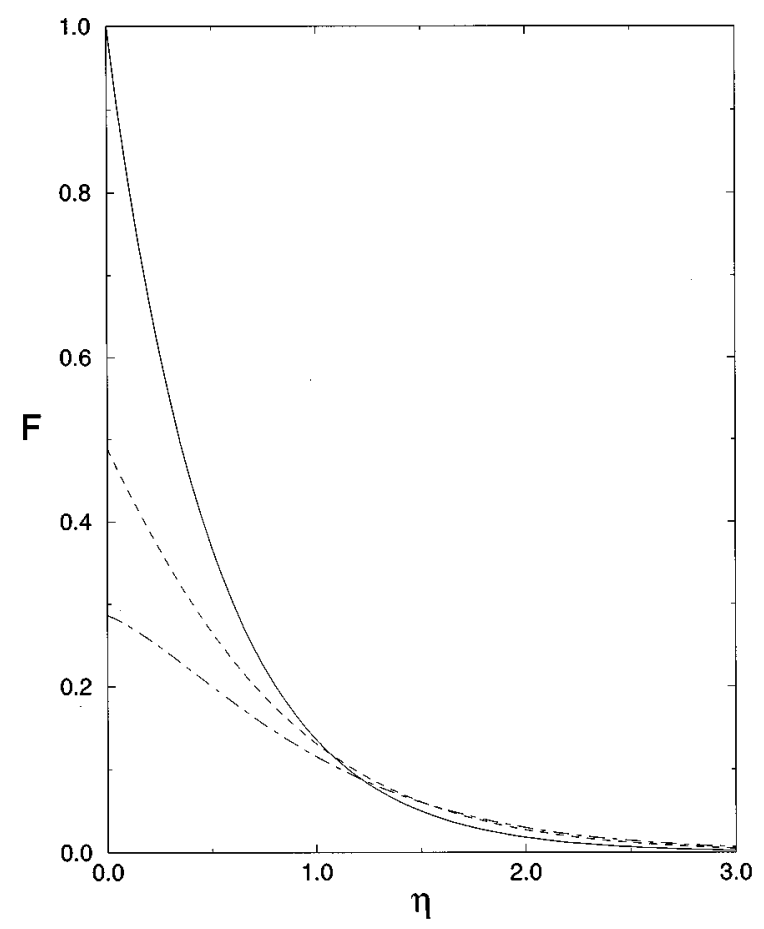

FIG. 1. The curves $F(\eta ; T)$ are presented for $T=0$ (top curve), $T=1.25$ (middle curve), and $T=2.0$ (bottom curve). Around $T=1.25$ the curve is changing from exponential (top curve) to Boltzmann (bottom curve) decay.

The function $G$, and hence $w$, is positive for all relevant values of its arguments. This is a consequence of the following Lemma, which is proved in the Appendix.

Lemma: The polynomials $S_{n}(x)$ are all positive for $x>0$. All of the thermal properties of the system will now be obtainable from the moments (note not pseudomoments) of $\eta$,

$$
\begin{aligned}
\left\langle\eta^{n}\right\rangle_{T} & =\int w\left(p_{\theta}, p_{\phi}, \theta, \phi ; T\right) \eta^{n} d p_{\theta} d p_{\phi} d \theta d \phi \\
& =\frac{2}{Z(T)} \int \eta^{n+1} G(\eta ; T) d \eta .
\end{aligned}
$$

In practice only the even moments are required, and these will all be identical, following the remarks made in the preceding section, with the standard expressions obtained by differentiating the partition function. We have computed the function $F(\eta ; T)$, as defined by Eq. (44), and the result is displayed in Fig. 1. In Fig. 2 we exhibit the density $\eta F(\eta ; T)$.

We remark that the asymptotic form of $F(\eta ; T)$ (see the Appendix for details) for large $T$ is, provided $\eta^{2} \ll T$,

$$
F(\eta ; T) \sim(2 T)^{-1} e^{-\eta^{2} / T},
$$

which is the Boltzmann distribution for a classical rigid rotator. We think it is noteworthy that this asymptotic behavior appears naturally, without any arguments about coarse graining or grouping of energy levels. 


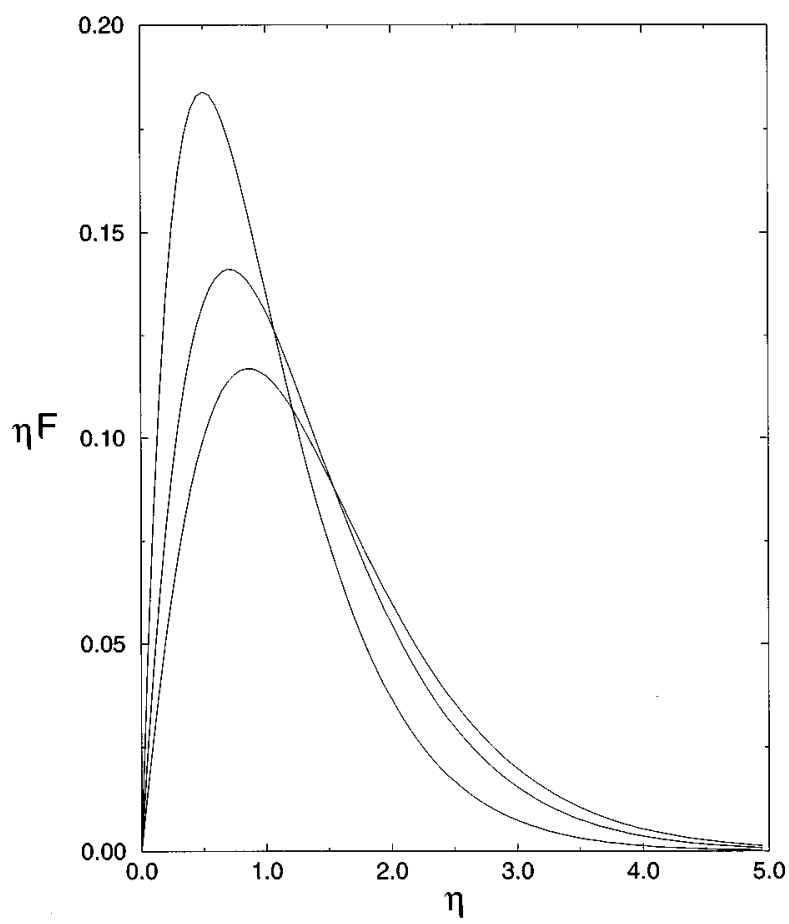

FIG. 2. The curves $\eta F(\eta ; T)$ are presented for $T=0$ (top curve), $T=1.25$ (middle curve), and $T=2.0$ (bottom curve).

\section{ACKNOWLEDGMENTS}

We thank our colleagues T. Recio and G. Trujillo of Universidad de Cantabria, T. Craven and G. Csordas of the University of Hawaii, and E.J. Watson of the University of Manchester for help with the Appendix. We acknowledge financial support of DGICYT Project No. PB-92-0507 (Spain). One of us (C.P.M.) also acknowledges partial financial support by CNPq (the Brazilian Research Council).

\section{APPENDIX}

For $T$ not too large the series representations (45) and (46), are an efficient means for the computation of Eq. (44), but for large $T$ the sum must be taken to increasingly large $\ell$, therefore it can be computed only in fast computers. Depending on the computer available, it then becomes more efficient to use an asymptotic series.

The basis for this series is an integral representation for $L_{\ell}$ obtained from the generating function (24), namely,

$$
\begin{aligned}
(-1)^{\ell} L_{\ell}(4 \eta) e^{-2 \eta}= & \frac{1}{2 \pi i} \int \frac{d z}{z^{\ell+1}(1+z)} \exp \left(-2 \eta \frac{1-z}{1+z}\right) \\
= & \frac{1}{2 \pi} \int_{-\frac{\pi}{2}+i \epsilon}^{\frac{\pi}{2}+i \epsilon} \operatorname{secs} \\
& \times \exp [2 i \eta \tan s-(2 \ell+1) i s] d s .
\end{aligned}
$$

Then, substituting in Eq. (45), and taking into account that $L_{\ell}$, as defined above, is zero for negative $\ell$,

$$
\begin{aligned}
G(\eta ; T)= & \pi^{-1} \int_{-\frac{\pi}{2}+i \epsilon}^{\frac{\pi}{2}+i \epsilon} d s \operatorname{secs} e^{2 i \eta \tan s} \\
& \times \sum_{\ell=-\infty}^{\infty} \exp \left[-\frac{\ell(\ell+1)}{T}-(2 \ell+1) i s\right] .
\end{aligned}
$$

The sum may be converted, by the Poisson sum formula, to obtain

$$
\begin{aligned}
G(\eta ; T)= & \sqrt{\frac{T}{\pi}} \exp \left(\frac{1}{4 T}\right) \int_{-\frac{\pi}{2}+i \epsilon}^{\frac{\pi}{2}+i \epsilon} d s \operatorname{secs} e^{2 i \eta \tan s} \\
& \times \sum_{n=-\infty}^{\infty}(-1)^{n} e^{-(s-n \pi)^{2} T} \\
= & \sqrt{\frac{T}{\pi}} \exp \left(\frac{1}{4 T}\right) \int_{-\infty+i \epsilon}^{\infty+i \epsilon} d s \operatorname{secs} e^{2 i \eta \tan s-s^{2} T} .
\end{aligned}
$$

We now obtain a uniform asymptotic expansion in $\eta$, for large $T$, by displacing the contour of Eq. (A3) to pass through the saddle point at $s=i \phi$, where

$$
\phi \cosh ^{2} \phi=\eta T^{-1} \text {. }
$$

We then obtain, by the method of steepest descents,

$$
\begin{aligned}
G(\eta ; T) \sim & \operatorname{sech} \phi(1+2 \phi \tanh \phi)^{-1 / 2} \\
& \times \exp \left[T\left(\phi^{2}-2 \phi \sinh \phi \cosh \phi\right)\right] .
\end{aligned}
$$

The asymptotic representation of $Z(T)$ follows particularly simply from the relation

$$
Z(T)=2 \int_{0}^{\infty} \eta G(\eta ; T) d \eta
$$

Substituting Eq. (A3) in Eq. (A6) we get, after one integration by parts,

$$
Z(T)=\sqrt{\frac{T^{3}}{\pi}} \exp \left(\frac{1}{4 T}\right) \int_{-\infty+i \epsilon}^{\infty+i \epsilon} s \exp \left(-s^{2} T\right) \operatorname{cosec} s d s .
$$

The power series expansion

$$
s \operatorname{cosec} s=1+\frac{1}{6} s^{2}+\frac{7}{360} s^{4}+\frac{31}{15120} s^{6}+\cdots
$$

then leads to

$$
Z(T) \sim T\left(1+\frac{1}{3} T^{-1}+\frac{1}{15} T^{-2}+\frac{4}{315} T^{-3}+\cdots\right) .
$$

The Lemma in Sec. IV may be proved by considering the generating function of $S_{n}$, which follows from that of $L_{\ell}$, in the form

$$
\sum_{n=0}^{\infty} S_{n}\left(4 x^{2}\right) z^{n}=[f(x, z)]^{2}
$$


where

where

$$
f(x, z)=\left(1-z^{2}\right)^{-1 / 2} \exp \left[\frac{2 z x^{2}}{1+z}\right] .
$$

Then, by constructing a linear, first-order partial differential equation for $f(x, z)$, it is possible to show that

$$
f(x, z)=\sum_{n=0}^{\infty} a_{n}(x) z^{n},
$$

and $H_{n}(x)$ are Hermite polynomials. Now, since the power series for $f(x, z)$ has positive coefficients for all real $x$, it follows that $[f(x, z)]^{2}$ has this same property, and hence the Lemma is proved.

[1] R. L. Hudson, Rep. Math. Phys. 6, 249 (1974).

[2] F. Soto and P. Claverie, J. Math. Phys. 24, 97 (1983).

[3] A. Einstein and O. Stern, Ann. Phys. 40, 551 (1913).

[4] P. Milonni, The Quantum Vacuum (Academic, San Diego, 1993), p. 28.

[5] J. Dalibard, J. Dupont-Roc, and C. Cohen-Tannoudji, J. Phys. (Paris) 43, 1617 (1982).

[6] P. Milonni, The Quantum Vacuum (Academic, San Diego, 1993), p. 142.

[7] T. W. Marshall, Proc. R. Soc. London, Ser. A 276, 475 (1963).
[8] H. M. França and T. W. Marshall, Phys. Rev. A 38, 3258 (1988).

[9] T. H. Boyer, in Foundations of Radiation Theory and Quantum Electrodynamics, edited by A. O. Barut (Plenum, New York, 1980).

[10] L. de la Peña and A. M. Cetto, The Quantum Dice. An Introduction to Stochastic Electrodynamics (Kluwer, Dordrecht, 1996).

[11] T. H. Boyer, Phys. Rev. A 18, 1228 (1978). 